\title{
Conhecimento e percepção dos pacientes sobre saúde bucal
}

\author{
Knowledge and perception of patients on oral health
}

\author{
Katia Simone Alves dos Santos* \\ Raquel Christina Barboza Gomes* \\ Ana Isabella Arruda Meira Ribeiro* \\ Darlene Cristina Ramos Eloy Dantas* \\ Camila Soares Sampaio** \\ Silmara Matias Augusto
}

\section{Resumo}

Objetivo: o objetivo desta pesquisa é avaliar o conhecimento e a percepção de saúde bucal pelos pacientes atendidos na Clínica de Periodontia da Universidade Estadual da Paraíba. Materiais e método: foi realizado um estudo longitudinal em uma amostra de 23 pacientes entre 13 e 62 anos de idade, com a aplicação de um questionário semiestruturado no início e no término do tratamento. Para as análises estatísticas foram utilizados testes de Qui-Quadrado de Pearson de medidas esperadas e observadas a fim de comparar os dados obtidos no pré e no pós-tratamento. Nas análises inferenciais, aceitou-se significância de $p \leq 0,05$. Resultados: dentre os principais resultados, verificou-se uma melhora na concepção da importância dos dentes ao término do tratamento, como também na autoavaliação de saúde bucal, que passou de regular para boa $(p<0,05)$. A frequência de escovação e uso do fio dental aumentou. $O$ conhecimento sobre a utilidade do flúor, do dentifrício e a duração dos dentes também teve uma frequência maior no pós-tratamento. Conclusão: observou-se que foi de grande importância a educação em saúde bucal pelos profissionais para a maior conscientização dos pacientes sobre a higiene oral, e assim concluiu-se que há necessidade de maior orientação sobre saúde bucal da parte dos alunos e profissionais da odontologia.

Palavras-chave: Conhecimento. Educação em saúde bucal. Percepção.

\section{Introdução}

A promoção da saúde deve englobar ações que envolvam todos os indivíduos, beneficiando assim as camadas mais diversas da população ${ }^{1}$. Quando os indivíduos são sujeitos de seu aprendizado, eles garantem a capacidade de identificar seus problemas e, dessa forma, conseguem intervir e impedir a evolução da doença. Logo, a prática de educação em saúde contribui, positivamente, na ampliação da autonomia, no cuidado e na promoção da saúde ${ }^{2}$, fornecendo aos usuários o controle saúde-doença e a condução de seus hábitos por meio da inserção de conhecimento ${ }^{3}$.

A literatura demonstra que os hábitos de higiene bucal, a presença de placa bacteriana e o sangramento gengival não são igualmente distribuídos na população e que variam de acordo com fatores sociodemográficos, como idade, gênero, nível econômico e cultural $^{4,5}$. Sabe-se ainda que mudanças de hábitos, muitas vezes, são difíceis de ser atingidas devido às influências sociais, culturais e governamentais que podem gerar uma verdadeira inversão de valores ${ }^{6}$.

O conceito de qualidade de vida está diretamente relacionado ao de autopercepção, no que diz respeito à interpretação das experiências e do estado de saúde geral do indivíduo no contexto da vida diária, e ambos são baseados nos conhecimentos sobre saúde

\footnotetext{
Professoras doutoras, Departamento de Odontologia da Universidade Estadual da Paraíba, Campina Grande, PB, Brasil.

Graduada em Odontologia, Departamento de Odontologia da Universidade Estadual da Paraíba, Campina Grande, Paraíba, PB, Brasil.

** Graduanda em Odontologia na Universidade Estadual da Paraíba, Departamento de Odontologia, Campina Grande, PB, Brasil.
} 
e doença ${ }^{7-9}$. Na maioria das vezes, a razão para as pessoas não procurarem atendimento odontológico é a não percepção de suas necessidades ${ }^{10}$. Ao avaliar sua condição bucal, os indivíduos usam critérios diferentes dos utilizados pelos profissionais, enquanto o cirurgião-dentista avalia a condição com base na ausência ou presença de doença, o paciente dá mais importância aos sintomas e problemas funcionais e sociais que são ocasionados pela presença da doença ${ }^{11}$.

A autoavaliação proporciona ao indivíduo o entendimento de sua própria condição bucal e é um fator essencial, tanto para que ele possa ficar motivado a buscar melhorias na saúde bucal como para que as estratégias preventivas e educativas possam ser idealizadas da melhor forma ${ }^{12}$.

A partir dessa concepção, o objetivo da pesquisa era avaliar os conhecimentos e a percepção sobre higiene e saúde bucal dos pacientes atendidos na Clínica de Periodontia da Universidade Estadual da Paraíba (UEPB), campus I, no município de Campina Grande, PB.

\section{Materiais e método}

Previamente à pesquisa, foi realizado um estudo piloto com dez pacientes atendidos na Clínica Odontológica de Periodontia da UEPB, com a aplicação de um questionário semiestruturado. Os sujeitos da pesquisa assinaram um termo de consentimento livre e esclarecido para participação no estudo.

Foi realizado um estudo longitudinal com 23 pacientes de ambos os gêneros e com idades entre 13 e 62 anos, atendidos entre os meses de agosto e novembro de 2012. A fim de garantir a representatividade, a amostra foi estratificada em duas fases. Sendo assim, os pacientes foram avaliados e questionados antes de iniciar o tratamento periodontal, e ao término foi reaplicado o questionário.

O questionário continha itens de identificação dos pacientes e, para caracterizar a amostra, perguntas objetivas e subjetivas referentes à temática. Ele foi aplicado individualmente aos participantes de forma reservada, evitando qualquer tipo de constrangimento e permitindo liberdade nas respostas.

Para a análise, os dados foram armazenados e tabulados no PASW, versão 21. Na descrição dos dados, foi utilizada a média como medida de tendência central, o desvio padrão (+) como medida de dispersão, além da exposição das frequências e respectivas porcentagens. Foi utilizado ainda testes de Qui-Quadrado de medidas esperadas e observadas, para comparar o pré e o pós-tratamento. Nas análises inferenciais, aceitou-se significância de $\mathrm{p} \leq 0,05$.

O estudo foi aprovado pelo Comitê de Ética em Pesquisa da Universidade Estadual da Paraíba sob o número do CAAE 06632912.8.0000.5187 e Parecer $\mathrm{n}^{\mathrm{o}}$ 238155, em concordância com a Resolução 196/1996 do Comitê Nacional de Saúde.

\section{Resultados}

Os dados obtidos no questionário foram organizados em tabelas de acordo com os itens de identificação dos pacientes e de caracterização da amostra. A Tabela 1 apresenta o grau de importância dado aos dentes antes e após o tratamento periodontal.

Tabela 1 - Grau de importância de diferentes variáveis associadas à saúde bucal no pré e pós-tratamento periodontal

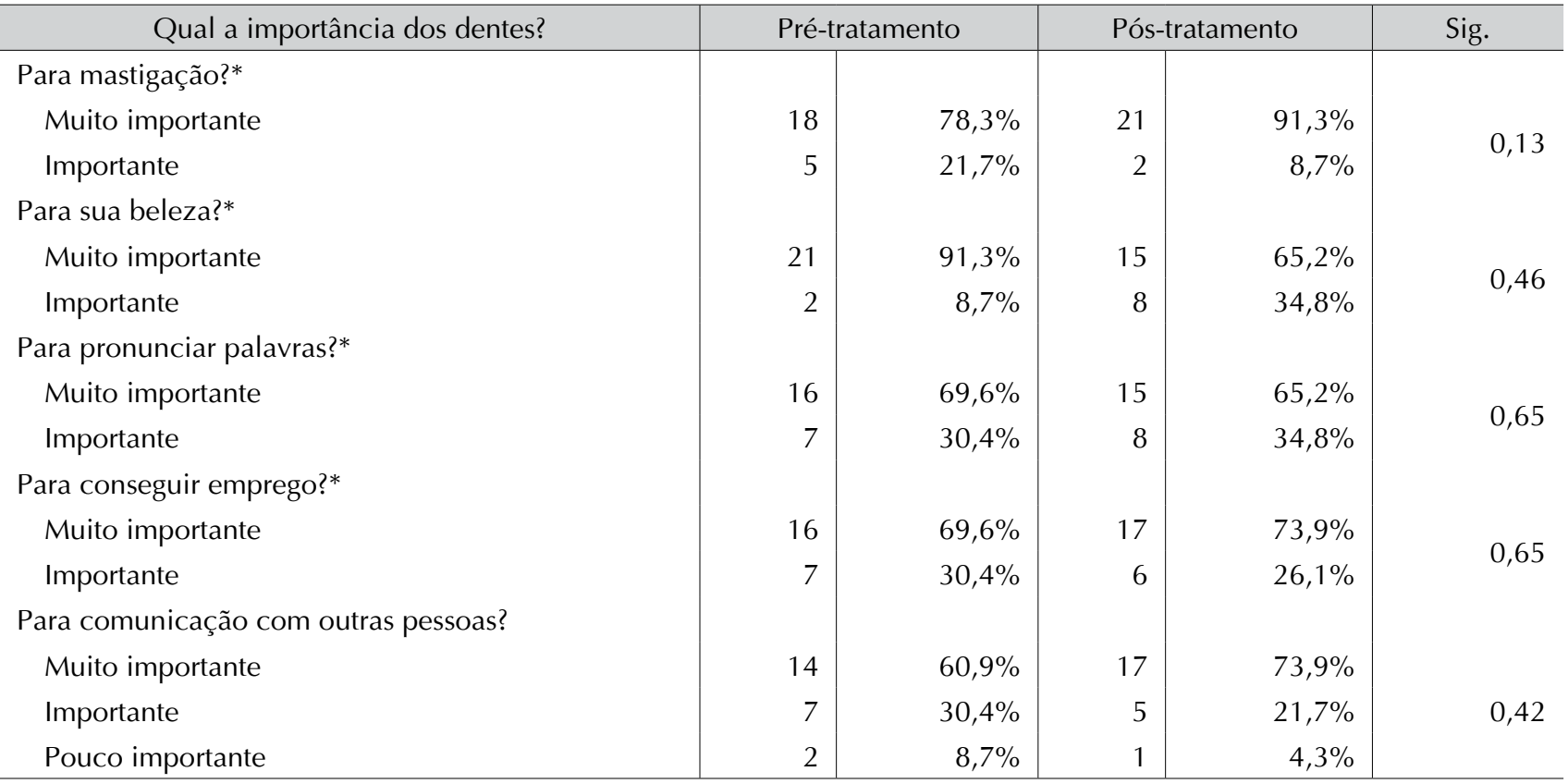

* Não tem frequência na alternativa Pouco importante.

Teste Qui-quadrado de Pearson

Fonte: todas as tabelas são de elaboração dos autores com base nos dados da pesquisa. 
Na Tabela 2, são apresentados os resultados da distribuição dos pesquisados segundo as questões de percepção e comportamento do paciente em relação à saúde bucal no pré e pós-tratamento periodontal.
Pôde-se observar que a maioria dos pacientes classificou sua saúde bucal como regular $(56,5 \%)$ antes do tratamento e boa $(52,2 \%)$ após o tratamento.

Tabela 2 - Distribuição dos pesquisados segundo as questões de percepção e comportamento do paciente em relação a saúde bucal no pré e pós-tratamento periodontal

\begin{tabular}{|c|c|c|c|c|c|}
\hline \multirow[b]{2}{*}{ Como você classificaria sua saúde bucal? } & \multicolumn{2}{|c|}{ Pré-tratamento } & \multicolumn{2}{|c|}{ Pós-tratamento } & \multirow[t]{2}{*}{ Sig. } \\
\hline & & & & & \\
\hline Ótima & 1 & $4,3 \%$ & 3 & $13 \%$ & \\
\hline Boa & 6 & $26,1 \%$ & 12 & $52,2 \%$ & \\
\hline Regular & 13 & $56,5 \%$ & 6 & $26,1 \%$ & 0,01 \\
\hline Ruim & 2 & $8,7 \%$ & 1 & $4,3 \%$ & \\
\hline Péssima & 1 & $4,3 \%$ & 1 & $4,3 \%$ & \\
\hline \multicolumn{6}{|l|}{ Porque você acha importante ter dentes saudáveis? } \\
\hline Para manter a saúde da boca & 10 & $43,5 \%$ & 10 & $43,5 \%$ & \\
\hline Para manter a saúde geral & 12 & $52,2 \%$ & 13 & $56,5 \%$ & * \\
\hline Para melhorar a aparência & 1 & $4,3 \%$ & 0 & 0 & \\
\hline \multicolumn{6}{|l|}{ Quantas vezes por dia você escova os dentes? } \\
\hline Uma & 1 & $4,3 \%$ & 3 & $13 \%$ & \\
\hline Duas & 7 & $30,4 \%$ & 0 & 0 & \\
\hline Três & 13 & $56,5 \%$ & 16 & $69,6 \%$ & * \\
\hline Quatro & 2 & $8,7 \%$ & 3 & $13 \%$ & \\
\hline Mais de quatro & 0 & 0 & 1 & $4,3 \%$ & \\
\hline \multicolumn{6}{|l|}{ Usa fio dental? } \\
\hline Sim & 11 & $47,8 \%$ & 16 & $69.6 \%$ & \\
\hline Não & 12 & $52,2 \%$ & 7 & $30,4 \%$ & \\
\hline \multicolumn{6}{|l|}{ Entre os que usam fio dental } \\
\hline Somente para limpar os dentes do fundo & 1 &, $1 \%$ & 0 & 0 & \\
\hline Somente para limpar os dentes da frente & 1 & $9,1 \%$ & 1 & $6,3 \%$ & \\
\hline Somente para remover restos de alimentos entre os dentes & 6 & $54,5 \%$ & 5 & $31,3 \%$ & \\
\hline Para remover os restos de alimento e a placa bacteriana entre os dentes & 3 & $27,3 \%$ & 10 & $62,5 \%$ & \\
\hline Não usa fio dental & & & & & \\
\hline Falta de hábito/esquecimento/falta de tempo & 7 & $58,3 \%$ & 3 & $42,8 \%$ & \\
\hline Não precisa & 2 & $16,7 \%$ & 2 & $28,6 \%$ & \\
\hline Descuido/preguiça & 3 & $25,0 \%$ & 2 & $28,6 \%$ & \\
\hline
\end{tabular}

${ }^{*}$ Com frequência zero em pelo menos uma célula.

Teste Qui-Quadrado de Pearson.

Algumas frequências não completaram 23 participantes, pois apresentaram missing. 
Os resultados referentes à distribuição dos pesquisados segundo as questões de conhecimento e orientação de saúde bucal no pré e pós-tratamento periodontal estão apresentados na Tabela 3.

Tabela 3 -Distribuição dos pesquisados segundo as questões de conhecimento e orientação de saúde bucal no pré e pós-tratamento

Qual a utilidade do flúor?

Deixar os dentes mais brancos

Impedir sangramento da gengiva

Evitar que o dente tenha cárie

Não é importante para os dentes

Não sabe

Motivos para utilizar pasta dental**

Para ajudar na limpeza

Para manter os dentes brancos

Para melhorar o hálito

O que o(a) senhor(a) conhece sobre a duração da dentição humana?

Os dentes duram a vida toda

Os dentes não duram a vida toda

Outros

Não sabe

Formas que adquiriu conhecimentos sobre saúde bucal?**

Com cirurgião-dentista

Por TV, rádio ou propagandas

Não tem conhecimento

Os profissionais da odontologia orientam sobre saúde bucal?

Sim

Não

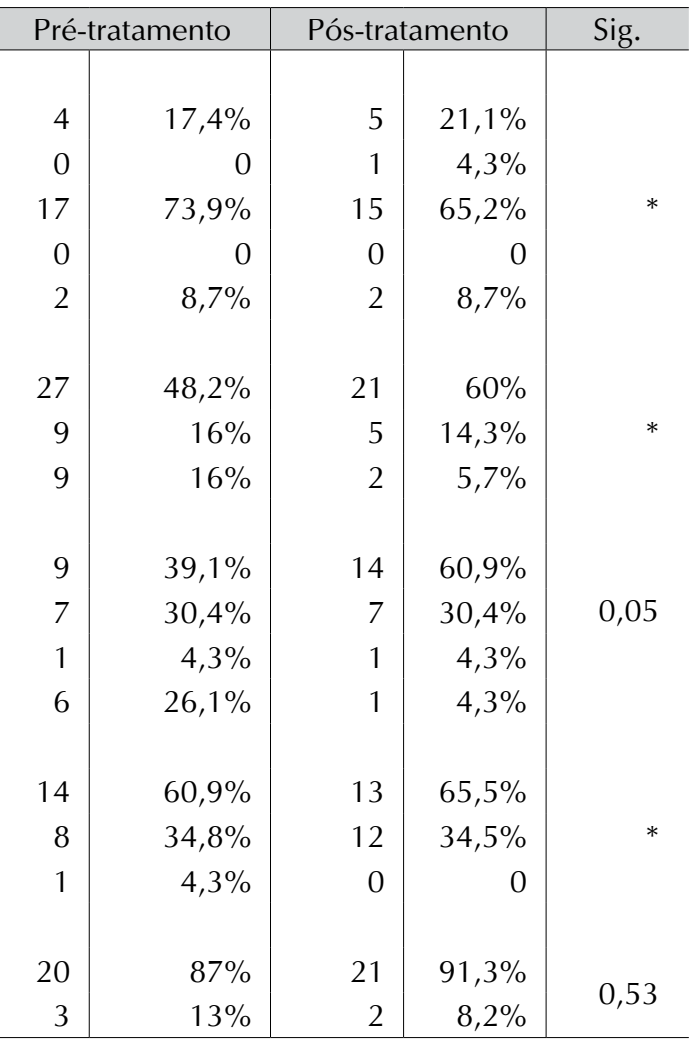

* Com frequência zero em pelo menos uma célula.

** Perguntas que permitiam mais de uma resposta.

Teste Qui-Quadrado de Pearson.

Algumas frequências não completaram 23 participantes, pois apresentaram missing.

Na Tabela 4, são apresentados os resultados da frequência e os tipos de problemas dentários e gengivais e a comparação no pré e pós-tratamento.

Tabela 4 - Distribuição dos pesquisados segundo as questões de percepção de problemas dentários e gengivais no pré e pós-tratamento periodontal

\begin{tabular}{|c|c|c|c|c|c|}
\hline & \multirow{2}{*}{\multicolumn{2}{|c|}{ Pré-teste }} & & & \multirow{3}{*}{ Sig. } \\
\hline & & & \multicolumn{2}{|c|}{ Pós-teste } & \\
\hline & $\mathrm{F}$ & $\mathrm{P}(\%)$ & $\mathrm{F}$ & $\mathrm{P}(\%)$ & \\
\hline \multicolumn{6}{|l|}{ Você tem algum problema com seus dentes? } \\
\hline Sim & 18 & $78,3 \%$ & 17 & $73,9 \%$ & \multirow{2}{*}{0,56} \\
\hline Não & 5 & $21,7 \%$ & 6 & $26,1 \%$ & \\
\hline \multicolumn{6}{|l|}{ Você tem algum problema com seus dentes? Quais? } \\
\hline Cárie/restaurações defeituosas & 7 & $38,9 \%$ & 8 & $44,4 \%$ & \multirow{6}{*}{0,02} \\
\hline Sensibilidade/dor de dente & 5 & $27,8 \%$ & 2 & $11,1 \%$ & \\
\hline Perda dentária/necessidade de prótese /má oclusão & 1 & $5,6 \%$ & 2 & $11,1 \%$ & \\
\hline Tratamento endodôntico & 2 & $11,1 \%$ & 1 & $2,9 \%$ & \\
\hline Cálculo/tártaro/fazer limpeza & 2 & $11,1 \%$ & 3 & $16,7 \%$ & \\
\hline Outros & 1 & $5,6 \%$ & 2 & $11,1 \%$ & \\
\hline \multicolumn{6}{|l|}{ Você tem algum problema com suas gengivas? } \\
\hline Sim & 10 & $43,5 \%$ & 13 & $56,5 \%$ & \multirow{2}{*}{0,2} \\
\hline Não & 13 & $56,5 \%$ & 10 & $43,5 \%$ & \\
\hline \multicolumn{6}{|l|}{ Você tem algum problema com suas gengivas? Quais? } \\
\hline Sangramento & 5 & $50 \%$ & 9 & $69,2 \%$ & \multirow{4}{*}{ * } \\
\hline Gengivite & 3 & $30 \%$ & 1 & $7,7 \%$ & \\
\hline Trauma gengival & 1 & $10 \%$ & 2 & $15,4 \%$ & \\
\hline Hiperplasia gengival & 1 & $10 \%$ & 0 & 0 & \\
\hline
\end{tabular}

* Com frequência zero em pelo menos uma célula. 


\section{Discussão}

A amostra deste estudo foi composta predominantemente por mulheres $(73,9 \%)$, concordando com vários estudos ${ }^{13-16}$ que constataram que as mulheres usam serviços de saúde mais frequentemente do que os homens. Isso reverte-se em uma situação favorável, uma vez que a mulher, quando conscientizada e adequadamente preparada, pode assumir o papel de principal agente de saúde na família ${ }^{17}$. Considerou-se importante classificar esses indivíduos segundo a renda, pois as doenças que afetam os indivíduos não podem ser explicadas somente pelos fatores biológicos que as caracterizam, visto que a qualidade de vida, decorrente de aspectos sociais, econômicos, políticos e culturais de uma sociedade, é um determinante essencial ${ }^{18}$. Portanto, observou-se neste estudo que a maioria dos sujeitos entrevistados $(39,1 \%)$ recebia entre um e três salários mínimos, seguido por zero ou um salário mínimo.

A caracterização social da amostra evidenciou que $60,9 \%$ dos participantes tinham idade entre 20 e 49 anos, com uma média de idade de 39,4 anos $( \pm 15,5)$. Atualmente, vivencia-se um quadro precário de saúde bucal da faixa etária adulta e idosa, resultado justamente da ausência de programas específicos para esse grupo populacional. Por isso, devido a esse abandono e às dificuldades enfrentadas, ações voltadas à educação em saúde, com ênfase na autoproteção e autopercepção, deveriam ser mais exploradas ${ }^{11}$.

Com base no que a Tabela 1 descreve sobre o grau de importância dado aos dentes antes e após o tratamento periodontal, nenhuma das variáveis apresentou diferenças estatisticamente significativas. Ainda assim, verificou-se que a maioria das pessoas avaliaram os dentes como sendo muito importantes para a mastigação (pré $=78,3 \%$, pós $=91,3 \%$ ) e para o aspecto beleza (pré $=91,3 \%$, pós $=65,2 \%$ ). Essa grande percentagem pode ser explicada pela crescente valorização da estética na odontologia contemporânea, tendo em vista que diariamente observa-se a valorização da beleza e da vaidade nas mais variadas práticas e artifícios ${ }^{19}$. Essas mudanças de hábitos comportamentais visam à maior inclusão social e ao bem-estar dos pacientes devido ao aumento do apelo estético que lhes é imposto no dia a dia ${ }^{20}$.

A Tabela 2 mostra que a maioria dos pacientes classificou sua saúde bucal como regular $(56,5 \%)$ antes do tratamento e como boa $(52,5 \%)$ após o tratamento, revelando uma melhora estatisticamente significativa $(p=0,01)$ na percepção de saúde bucal ao longo do tratamento. Outras pesquisas ${ }^{11,17,18}$ também encontraram uma autoavaliação de saúde bucal regular no início do tratamento.

Embora, no presente estudo não tenha sido feito o exame clínico dos participantes e, portanto, não tenha sido possível comparar esses dados subjetivos com a situação clínica, estudos sobre autoper- cepção $0^{21}$ já mostraram que a maioria das pessoas vê sua condição bucal de maneira favorável mesmo com condições clínicas não satisfatórias, provavelmente porque as medidas clínicas de saúde utilizadas pelos profissionais são preceptores relativamente fracos da percepção de saúde bucal das pessoas.

Alguns autore ${ }^{21,22}$ mostraram que existe relação entre a percepção da condição bucal e algumas variáveis clínicas, mas que se trata de uma associação relativamente fraca. Uma das razões para essa fraca associação entre as variáveis clínicas e a autoavaliação deve-se ao fato de que muitas das doenças detectadas no exame clínico são assintomáticas e provavelmente desconhecidas do indivíduo ${ }^{23}$.

A variável "Por que você acha importante ter dentes saudáveis?" manteve-se praticamente constante após o tratamento, com uma pequena maioria assinalando o item "Para manter a saúde geral" (pré $=52,2 \%$, pós $=56,6 \%$ ). Esse resultado vai de encontro às afirmações de outras pesquisas ${ }^{24,25}$. Ainda assim, vale ressaltar a grande percentagem de pacientes que ainda tem a concepção de que problemas dentários estão relacionados apenas com a saúde bucal, evidenciando-se a necessidade de melhoria das informações que são passadas para a população em geral.

Em relação à frequência de escovação diária, houve um pequeno aumento no número de escovações do grupo que escova três e quatro vezes ao dia, revelando maior atenção à higiene bucal após o tratamento e corroborando com os achados de outros estudos $^{18,26}$. No que se refere à maior frequência de escovação, ela é importante porque funciona como uma aplicação tópica de flúor diária, por meio do uso de dentifrícios fluoretados ${ }^{27}$; quanto à escovação dos dentes três vezes ao dia, é importante para a prevenção de doenças bucais ${ }^{28}$.

Ainda, na Tabela 2, observou-se a descrição dos pacientes que utilizam fio dental e quais os motivos. Verificou-se que houve um aumento na utilização de fio dental após o tratamento (pré $=47,8 \%$, pós $=69,6 \%)$. A maioria $(54,5 \%)$ relatou utilizar fio dental para remover restos de alimentos entre os dentes. Entretanto, aqueles que relataram não utilizar fio dental justificaram por falta de hábito/ esquecimento ou falta de tempo (58,3\%). Esse resultado provavelmente está associado à orientação repassada pelos alunos ao longo do tratamento. A crença de que o fio dental tem como função a remoção de restos alimentares entre os dentes estimula sua utilização com pequena frequência, pois os pacientes somente farão uso dele quando comerem alimentos sólidos e se esses ficarem entre os dentes. Essa atitude incorreta deve ser modificada, mediante esclarecimentos sobre a verdadeira função do fio dental ${ }^{29}$.

A escovação convencional não é efetiva para a higienização da região interproximal. Ainda, tendo-se por base que, mais frequentemente a doença periodontal inicia-se nessa área, a remoção de placa 
bacteriana da região interproximal, além de prevenir a cárie, é de especial importância para prevenir a doença periodontal ${ }^{17}$.

Em relação à prevenção da cárie dentária, a utilização do flúor é reconhecidamente o método mais recomendado e utilizado pelos profissionais da área odontológica. Verificou-se que a maioria dos participantes (pré $=73,9 \%$, pós $=65,2 \%$ ) reconheceu no uso do flúor uma medida para controlar a doença, fato também constatado em outros estudos ${ }^{18,28,30-31}$.

Para a variável que avaliou o motivo da utilização do creme dental, constatou-se um maior número de respostas para o item "Para ajudar na limpeza" (pré $=48,2 \%$, pós $=60 \%$ ). Em uma visão mais ampla, o dentifrício foi definido como tudo aquilo que é usado com a escova de dente para remover restos de alimentos e ainda conferir bom hálito ao usuário ${ }^{32}$. Nesta pesquisa, apenas $16 \%$ dos pacientes associaram o uso do dentifrício à "melhora do hálito" no pré-tratamento, e essa percentagem diminuiu pra 5,7\% no pós-tratamento. Deve ficar claro que o dentifrício não é essencial para a remoção de placa dentária. Ele apenas auxilia na limpeza dos dentes e, no caso do dentifrício fluoretado, ajuda na prevenção de cárie dental ${ }^{33}$.

Com relação às percepções dos entrevistados sobre a conservação dos dentes, foi encontrada uma diferença estatisticamente significativa $(p=0,05)$ entre os questionários iniciais e finais. Houve um aumento considerável entre aqueles que disseram que os dentes duram a vida toda (pré $=39,1 \%$, pós $=60,9 \%$ ), possivelmente, esse aumento deve-se à redução no grupo que afirmou não ter conhecimento prévio ao tratamento.

Observou-se que, embora a maioria dos participantes acredite que os dentes possam durar a vida toda, isso é condicionado à responsabilidade individual pelo autocuidado e à procura pelo dentista. $\mathrm{O}$ fato de que $30,4 \%$ não acreditam na durabilidade dos dentes ao longo da vida pode estar relacionado a situações próprias vividas por essas pessoas, como a perda de dentes, ou por presenciarem o fato em pessoas próximas. Outros trabalhos na literatura também constataram a mesma situação $0^{34-36}$.

Quanto à aquisição de conhecimentos sobre saúde bucal, a maioria dos participantes (pré $=60,9 \%$, pós $=65,5 \%$ ) apontou o cirurgião-dentista como principal fonte de informações sobre saúde bucal, demonstrando a importância da classe odontológica quanto à educação com vistas à manutenção da saúde bucal da população. Pesquisas realizadas em diferentes países ${ }^{37-39}$ também encontraram o cirurgião-dentista como principal fonte de informações, representando $57 \%, 90 \%, 82,3 \%$ da população estudada, respectivamente.

Quando perguntados se “Os profissionais da odontologia orientam sobre saúde bucal?", não foi encontrada nenhuma diferença estatisticamente significativa $(\mathrm{p}=0,53)$ entre pré e pós-teste, sendo assim, a maioria dos participantes permaneceu no grupo que afirmou que os dentistas fazem orientação sobre saúde bucal (pré $=87 \%$, pós $=91,3 \%$ ). De acordo com alguns autores ${ }^{40,41}$, a função de educador do cirurgião-dentista é tão importante quanto o domínio das técnicas curativas. A odontologia dos tempos modernos, baseada em atitudes e tratamentos preventivos, deve permitir que o profissional esteja apto a motivar e educar o paciente para a prevenção de cáries e doença periodontal.

A Tabela 4 demonstrou a frequência e os tipos de problemas dentários e gengivais e a comparação entre o pré e o pós-tratamento. A maioria (pré = 78,3\%, pós $=73,9 \%$ ) relatou ter problemas com os dentes. Sendo que $38,9 \%$ no pré e $44,4 \%$ no pós-tratamento disseram ter problemas com cárie ou restaurações defeituosas. Foi encontrada uma diferença significativa de frequência entre o pré e o pós-tratamento $(\mathrm{p}=0,02)$. Esse índice elevado pode estar relacionado ao fato de que, geralmente, o paciente não conhece ou não dá importância às alterações bucais que não sejam cáries ou a doença periodontal, nem mesmo ao autoexame para identificá-las, devido à falta de divulgação de seus sinais e sintomas característicos. Cericato e Lamha ${ }^{42}$ (2012) enfatizaram, em seu estudo, a necessidade de esclarecimento da população acerca do processo saúde-doença bucal, destacando a possibilidade de intervenção precoce e de controle dos problemas de saúde. Esses autores mostraram também que um dos métodos citados para prevenir a doença é o autocuidado.

Verificou-se que $43,5 \%$ dos pacientes que compuseram a amostra acreditavam ter algum tipo de problema na gengiva no pré-teste; no pós-teste, esse índice aumentou para 56,5\%. Esse padrão de resposta provavelmente está relacionado à falta de consciência dos problemas gengivais dos pacientes antes do tratamento. $\mathrm{O}$ problema mais frequente foi o sangramento (pré $=50 \%$, pós $=69,2 \%$ ), não tendo sido encontrada nenhuma diferença estatisticamente significativa entre o pré e o pós-tratamento.

Logo, é de fundamental importância a elaboração de estratégias para controle e redução de doenças na população por meio do incentivo à promoção de saúde e da participação do indivíduo no processo de aprendizado no intuito de melhorar a qualidade de vida geral do indivíduo.

\section{Conclusão}

Embora os pacientes atendidos na clínica de periodontia tenham apresentado atitudes positivas, bom conhecimento e boa percepção sobre saúde bucal, os resultados observados quanto à aquisição de conhecimento durante o tratamento demonstrou que ainda há necessidade de uma maior orientação de parte dos discentes. 


\section{Abstract}

Objective: This research aimed to assess the knowledge and perception on oral health by patients assisted at the Periodontics Clinic of the Paraíba State University. Methods: A longitudinal study was performed with a sample of 23 patients aged 13-62 years, and a semistructured questionnaire was applied at the beginning and end of treatment. For statistical analysis, Pearson's chi-square test on expected and observed measures was used in order to compare data obtained at pre- and posttreatment. The inferential analysis accepted significance of $p \leq 0.05$. Results: Among the main results, it was verified an improvement of the concept of teeth importance after treatment, as well as of oral health self-assessment, which went from "average" to "good" $(p<0.05)$. Brushing frequency and flossing increased. The knowledge on fluoride and dentifrice use, and teeth duration also presented a higher frequency at post-treatment. Conclusion: It was observed the major importance of oral health education performed by professionals to increase the awareness of patients regarding oral hygiene, and so it was concluded that further guidance on oral health, by students and Dentistry professionals, was required.

Keywords: Knowledge. Oral health education. Perception.

\section{Referências}

1. Teles MS, Groisman S. Promoção de saúde bucal através da educação a distância. Perionews 2012; 6(4):435-9.

2. Silva ASCd, Rizzante FAP, Picolini MM, Campos Kd, Corrêa CdC, Franco EC et al. Bauru School of Dentistry TeleHealth League: an educational strategy applied to research, teaching and extension among applications in tele-health. J Appl Oral Sci 2011; 19(6):599-603.

3. Alves VS. Um modelo de educação em saúde para o Programa Saúde da Família: pela integralidade da atenção e reorientação do modelo assistencial. Interface - Comunic Saúd Educ 2004/2005; 9(16): 39-52.

4. Al-Otaibi M, Angmar-Mansson B. Oral hygiene habits and oral health awareness among Urban Saudi Arabians. Oral Health Prev Dent 2004; 2(4):389-96.

5. Almas K, Al-Hawish A, Al-Khamis W. Oral hygiene practices, smoking habit, and self-perceived oral malodor among dental students. J Contemp Dent Pract 2003; 4(4):77-90.

6. Arcieri RM, Rovida TAS, Lima DP, Garbin AJI, Garbin CAS. Análise do conhecimento de professores de educação infantil sobre saúde bucal. Educ Rev 2013; 47(1):301-14.

7. Bombarda-Nunes FdF, Miotto MHMdB, Barcellos LA. Autopercepção de saúde bucal do agente comunitário de saúde de Vitória, ES, Brasil. Pesq Bras Odontoped Clin Integr 2008; 8(1):7-14.

8. Martins AMEBL, Barreto SM, Silveira MFd, Santa-Rosa TTdA, Pereira RD. Self-perceived oral health among Brazilian elderly individuals. Rev Saúde Públ 2010; 44(5):912-22.

9. Matos IDL, Lima-Costa MF. Auto-avaliação da saúde bucal entre adultos e idosos residentes na Região Sudeste: resultados do Projeto SB-Brasil, 2003. Cad Saúde Pública 2006; 22(8):1699-707.

10. Silva SRCdS, Fernandes RAC. Autopercepção das condições de saúde bucal por idosos. Rev Saúde Púb 2001; 35(4):1-10.
11. Bortoli D, Locatelli FA, Fadel CB, Baldani MH. Associação entre percepção de saúde bucal e indicadores clínicos e subjetivos: estudo em adultos de um grupo de educação continuada da terceira idade. UEPG Ci Biol Saúde 2003; 9(3/4):55-65.

12. Rosa RR, Henriques JCG, Anhalt ACF, Castilho JCdM, Rodrigues JR, Nicodemo D. Autopercepção da saúde bucal e anamnese em idosos. Rev Cienc Med 2013; 22(1):5-11.

13. Dias NMDO. Mulheres: sanitaristas de pés descalços. São Paulo: Hucitec; 1991.

14. Giron MCC, Oliveira JP, Klein HJ, Schutz R, Pia R. Estudo sobre a consulta preventiva em odontologia: realidade e sugestão. Rev Odonto Cienc 1986; 1(1):58-71.

15. Queiroz MS. Representações sobre saúde-doença: agentes de cura e pacientes no contexto do SUDS. Campinas: Unicamp; 1991.

16. Vasconcellos MCC, Silveira F. Conhecimento sobre a manutenção da própria saúde bucal em população que demanda centro de saúde. Rev Odontol Unesp 1989; 18(1):225-32.

17. Unfer B, Saliba O. Avaliação do conhecimento popular e práticas cotidianas em saúde bucal. Rev Saúde Pub 2000; 34(2):190-5.

18. Silva SRC, Rosell LF, Valsecki Júnior A. Percepção das condições de saúde bucal por gestantes atendidas em uma unidade de saúde no município de Araraquara, São Paulo, Brasil. Rev Bras Saúde Matern Infant 2006; 6(4):405-10.

19. Aimi E, Lopes GC. Restaurações diretas de resina composta em dentes posteriores: uma realidade no Brasil do século XXI. Clin Int J Braz Dent 2007; 3(1):32-40.

20. Botta AC, Duarte SJr, Paulin FPI, Gheno SM, Powers JM. Surface roughness of enamel and four resin composites. Am J Dent 2009; 22(5):252-4.

21. Atchison KA, Dolan TA. Development of the Geriatric Oral Health Assessment Index. J Dental Educ 1990; 54(1):680-7.

22. Steele JG, Walls AWG, Ayatollahi SMT, Murray JJ. Major clinical findings from a dental survey of elderly people in three different English communities. Br Dent J 1996; 180(1):17-23.

23. Jokovic A, Locker D. Dissatisfaction with oral health status in an older adult population. J Public Health Dent 1997; 57(1):40-7.

24. Paes AMC, Rose MCP. Avaliação dos normalistas da escola normal de Ceilândia em educação em saúde bucal. Rev Saúde 1997; 8(3):32-43.

25. Martins EM. Educação em saúde bucal: os desafios de uma prática. Cad Odontol 1998; 1(2):30-40.

26. Menezes DR, Cavalcanti AL. Estudo comparativo da informação em saúde bucal entre estudantes de cursos da área de saúde. Pesq Bras Odontoped Clin Integr 2003; 3(2):27-33.

27. Maltz M, Carvalho J. Tratamento da doença cárie. In: Kriger L (Org.). Promoção de saúde bucal. São Paulo: Artes Médicas; 1997. p. 95-111.

28. Naressi WG, Moreira EJG. Odontologia preventiva: agentes mecânicos de higiene bucal. Rev Gaúcha Odontol 1982; 30(2):154-6.

29. Santos PA, Rodrigues JA, Garcia PPNS. Conhecimento sobre a prevenção de cárie e doença periodontal e comportamento de higiene bucal de professores de ensino fundamental. Cienc Odontol Bras 2003; 6(1):67-74.

30. Petersen PE, Mzee MO. Oral health profile of schoolchildren, mothers and schoolteachers in Zanzibar. Community Dent Health 1998; 15(4):256-62.

31. Lawderi JAC, Mendes YBE, Silva LC, Andrade DKD, Rocha LM, Rogalla TM et al. Conhecimento e práticas em saúde bucal entre usuários de serviços odontológicos. Pesq Bras Odontoped Clin Integr 2008; 8(3):321-6. 
32. Cury JA. Dentifrícios: como escolher e como indicar. In: APCD. (Org.). Odontologia. São Paulo: Artes Médicas - Divisão Odontológica; 2002. v. 4. p. 281-295.

33. Kirchner UL, Mendonça LL, Costa RN. Higienização bucal. Educação para saúde bucal: manual para o ensino na escola de 1‥ Grau. Belo Horizonte: Editora UFMG/ISHIS; 1992. p. 63-73.

34. Bernd B, Souza CB, Lopes CB, Pires Filho FM, Lisboa IC, Curra LCD et al. Percepção popular sobre saúde bucal: o caso das gestantes do Valão. Saúde Debate 1992; (34):33-9.

35. Misrachi CL, Sáez MS. Valores, creencias y practicas populares en relación a la salud oral. Cuad Méd Soc 1989; 30(1):27-33.

36. Rocha MCBS. Avaliação do conhecimento e das práticas de saúde bucal: gestantes do Distrito Sanitário Docente-Assistencial Barra/RioVermelho - Município de Salvador, BA [Tese de Doutorado]. São Paulo: Faculdade de Odontologia da Universidade de São Paulo; 1993.

37. Glasrud PH, Frazier PJ. Future elementary schoolteachers' knowledge and opinions about oral health and community programs. J Public Health Dent 1988; 48(2):74-80.

38. Loupe MJ, Frazier PJ. Knowledge and attitudes of schoolteachers toward oral health programs and preventive dentistry. J Am Dent Assoc 1983; 107(2):229-34.

39. Lang P, Woolfolk MW, Faja BW. Oral health knowledge and attitudes of elementary schoolteachers in Michigan. J Public Health Dent 1989; 49(1):44-50.

40. Garcia PPNS, Pinto LC, Bonan RF. Avaliação de retorno periódico em escolares. Stoma 2000; 57(1):15-20.

41. Garcia PPNS, Dinelli W, Serra MC, Terence RL. Motivação do paciente para retorno: a chave para o sucesso do tratamento odontológico. J Assessor Odontol 1999; 3(17):39-42.

42. Cericato GO, Lamha APSF. Hábitos de saúde bucal de portadores de deficiência visual no contexto da saúde coletiva. Rev Fac Odontol Univ Passo Fundo 2012; 17(2):137-44.

\section{Endereço para correspondência:}

Katia Simone Alves dos Santos

José de Holanda, 561/204

50710-140 Torre Recife-PE

Telefone: (81) 3071-7036 / 99927-6591

E-mail: ksasantos@hotmail.com

Recebido: 03/11/14. Aceito: 23/10/15. 